American Journal of Applied Sciences 9 (9): 1457-1463, 2012

ISSN 1546-9239

(C) 2012 Science Publication

\title{
Moringa Oleifera Lam Mitigates Oxidative Damage and Brain Infarct Volume in Focal Cerebral Ischemia
}

\author{
${ }^{1,3}$ Woranan Kirisattayakul, ${ }^{2,3}$ Jintanaporn Wattanathorn, \\ ${ }^{2,3}$ Terdthai Tong-Un, ${ }^{2,3}$ Supaporn Muchimapura and ${ }^{2,3}$ Panakaporn Wannanon \\ ${ }^{1}$ Department of Physiology and Graduate School (Neuroscience Program), \\ Faculty of Medicine, \\ ${ }^{2}$ Department of Physiology, Faculty of Medicine, \\ ${ }^{3}$ Integrative Complementary Alternative Medicine Research and Development Group, \\ Khon Kaen University, Khon Kaen 40002, Thailand
}

\begin{abstract}
Problem statement: At present, the therapeutic outcome of cerebral ischemia is still not in the satisfaction level. Therefore, the preventive strategy is considered. Based on the protective effect against oxidative damage of Moringa Oleifera Lam. Leaves extract, we hypothesized that this plant extract might protect against cerebral ischemia, one of the challenge problems nowadays. In order to test this hypothesis, we aimed to determine the protective effect of M.oleifera leaves extract in animal model of focal cerebral ischemia induced by permanent occlusion of right middle cerebral artery. Approach: Male Wistar rats, weighing 300-350 g, were orally given the extract once daily at doses of 100,200 and $400 \mathrm{mg} \mathrm{kg}^{-1} \mathrm{BW}$ at a period of 2 weeks, then, they were permanently occluded the right Middle Cerebral Artery (MCAO). The animals were assessed the cerebral infarction volume and oxidative damage markers including MDA level and the activities of SOD, CAT and GSHPx enzymes at $24 \mathrm{~h}$ after occlusion. Results: Rats subjected to M.oleifera extract at all doses used in this study significantly decreased brain infarct volume both at cortical and subcortical structures in accompany with the elevation of SOD activity in both hippocampus and striatum while only the rats exposed to the extract at doses of 100 and $400 \mathrm{mg} \mathrm{kg}^{-1}$ BW showed the increased GSHPx activity in hippocampus. No the changes were observed. Therefore, our results demonstrates the potential benefit of M.oleifera leaves to decrease oxidative stress damage and brain infarct volume. Conclusion: This study is the first study to demonstrate the neuroprotective effect against focal cerebral ischemia of M.oleifera leaves. It suggests that M.oleifera may be served as natural resource for developing neuroprotectant against focal cerebral ischemia. However, the precise underlying mechanism and possible active ingredient are still required further study.
\end{abstract}

Key words: Moringa Oleifera, neuroprotective, cerebral ischemia, oxidative damage, brain infarct volume

\section{INTRODUCTION}

To date, ischemic stroke has been recognized as one of the challenge problems leading to both mortality and disability especially in the developing countries (Feigin, 2007). Despite the advances of technology, the therapeutic outcome of this condition is still not in the satisfaction level. The successful of treatment appears to depend on the treatment within the first 3 hours of symptom onset. Therefore, stroke prevention is considered.

Since oxidative stress damage is one of the earliest mechanisms responsible for tissue demise after cerebral ischemic insult (Warner et al., 2004), antioxidants have been proposed to be the promising agent for treating ischemic stroke (Margaill et al., 2005). Numerous substances possessing antioxidant activity have demonstrated the neuroprotective effect against cerebral ischemia in animal model of focal cerebral ischemia induced by permanent occlusion of right Cerebral Artery (MCAO) (Wattanathorn et al., 2011).

Moringa Oleifera Lam., a plant in a family of Moringaceae, is a multipurpose tree found almost all over the Asian countries including in Thailand. Its leaves are consumed not only as food but also as medicine. It has been shown to have anti-inflammatory, hypotensive (Caceres et al., 1992; Faizi et al., 1995) and nootropics (Mohan et al., 2005) activities. In

Corresponding Authors: Jintanaporn Wattanathorn, Department of Physiology, Faculty of Medicine, Khon Kaen University, Khon Kaen, Thailand, 40002 Tel: +66-43-348394 Fax: +66-43-348394 
addition, it also exhibits the protective effect against oxidative damage (Sreelatha and Padma, 2011) and antioxidant effect (Kumar and Pari, 2003). Since M.oleifera leaves are consumed as food, the chance of toxicity is very less. It has been reported that $\mathrm{LD}_{50}$ of alcoholic extract of M.oleifera leaf is approximate $2.8 \mathrm{~g}$ $\mathrm{kg}^{-1}$ BW (Sudha et al., 2010). Therefore, it is quite safe even when consumed in a higher quantity due to its high LD 50. Based on the crucial role of oxidative stress on the pathophysiology of cerebral ischemia and the protective effect against oxidative damage of M.oleifera, the neuroprotective effect of this plant extract is considered. Therefore, this study aimed to determine the effect of M.oleifera leaves extract on the oxidative stress markers including Malondialdehyde (MDA) level and the activities of Superoxide Dismutase (SOD), Catalase (CAT) and glutathione peroxidase (GSHPx) and the brain infarct volume both in cortical and subcortical structures.

\section{MATERIALS AND METHODS}

Experimental animals: Healthy male Wistar rats (300$350 \mathrm{gm}$ ) were obtained from National Animal Center, Salaya, Nakorn Pathom. They were randomly housed 5 per cage and maintained in 12:12 light: Dark cycle and given access to food and water ad libitum. The experiments were performed to minimize animals suffering and the experiment protocols were approved by the Institutional Animal Care and Unit Committee Khon Kaen University, Thailand.

Plant material: The fresh Moringa Oleifera Lam (Moringaceae) were harvested during November to December, 2010 from the Khon Kaen province Thailand. The plant specimen was authenticated by Associate Professor Dr. Panee Sirisa-ard, Faculty of Pharmaceutical Sciences, Chiangmai University, Thailand. The voucher specimen was kept at Integrative Complimentary Alternative Medicine Research and Development, Khon Kaen University, Khon Kaen, Thailand.

Plant material preparation: The fresh leaves were immediately cleaned, than cut into small pieces and dried at the temperature less than $50^{\circ} \mathrm{C}$. The dried plant material was ground into fine coarse powder and extracted with $50 \%$ alcoholic. After that evaporation of solvent in rotary evaporator affords a crude extract of the soluble components and filtrate was lyophilized. The percent yield of extract was $17.49 \%$. The extract contained total phenolic compounds at concentration of 86.73-93.6 $\pm 0.51 \mathrm{mg}$ of GAE. $\mathrm{g}^{-1}$ extract. The extracts were stored at $-25^{\circ} \mathrm{C}$ in a dark bottle until used. The crude extract was suspended in 1\% CMC (Sodium carboxymethylcellulose).

Focal cerebral ischemic induction: Animals were induced food deprivation at a period of 12 hours before surgery whereas water was allowed to assess. Then, they were anesthesized by injecting thiopental sodium at dose of $60 \mathrm{mg} \mathrm{kg}^{-1}$ body weight via intraperitoneal route. After the animals were anesthesized, the focal cerebral ischemic induction was performed. In brief, through a ventral midline incision, the bifurcation of right common carotid artery was exposed. The internal carotid artery and external carotid artery were distally dissected free from the adjacent tissues and ligated. The silicone-coated nylon monofilament was gently advanced passed up through the lumen of the internal carotid artery via arteriectomy in common carotid artery approximate $17 \mathrm{~mm}$ from the carotid bifurcation and advanced to the Circle of Willis to occlude the origin of the right middle cerebral artery. Then, the distal end of monofilament was tied up and the wound was suture.

Determination of infarction volume: Animals were sacrificed $24 \mathrm{~h}$ after right middle cerebral artery occlusion, the brain was immediately removed and prepared as coronal sections at $2 \mathrm{~mm}$ thick and immersed in 2\% 2, 3, 5-Triphenyltetrazolium Chloride (TTC) solution at a $15 \mathrm{~min}$ period. Morphometric measurement was performed using Image analyzer (Image Pro Plus 3 ). Total infarct volume for each brain was calculated by summation of the infracted area of all brain slices (area of infarct in square millimetersxthickness $(2 \mathrm{~mm})$ ) from the same hemisphere.

Determination of malondialdehyde: The brain regions including cortex, hippocampus and striatum were isolated and prepared as brain homogenate with $1.15 \% \mathrm{KCL}$. The Malondialdehyde (MDA) level in brain homogenate sample was determined by using thiobarbituric acid reaction (Tong-Un et al., 2010). Briefly, the following reagents including $100 \mu \mathrm{L}$ of $8.1 \%$ sodium dodecyl sulfate, $750 \mu \mathrm{L}$ of $20 \%$ acetic acid (pH 3.5), $750 \mu \mathrm{L}$ of $0.8 \%$ thiobarbituric acid and $300 \mu \mathrm{L}$ of distilled water were added to $100 \mu \mathrm{L}$ of brain homogenate sample in glass tube. The mixture was mixed and heated in water bath at $95^{\circ} \mathrm{C}$ for $1 \mathrm{~h}$. After cooling, $500 \mu \mathrm{L}$ of distilled water and the mixture of nbutanol: Pyridine (15:1) were added. The test tubes were centrifuged at $4,000 \mathrm{~g}$ for $10 \mathrm{~min}$ and the organic layer was taken to measure the absorbance at $532 \mathrm{~nm}$ with spectrophotometer (Pharmacia LKB-Biochrom 4060). 1, 1, 3, 3-Tetramethoxy Propane (TMP) at 
concentrations of ranging between 2-20 nmol were used as standard calibration curve. The results were normalized with protein concentration which determined and expressed as nmol $\mathrm{mg}^{-1}$ protein (Lowry et al., 1951).

Determination of superoxide dismutase (SOD): The activity of SOD in brain homogenate sample was estimated by using the xanthine/xanthine oxidase system for generating superoxide anion and measuring the reduction of cytochrom c (colorimetric reaction) as a scavenging activity of SOD via reader (Bio Rad model 680) at $550 \mathrm{~nm}$ (Kakkar et al.,1984). The activity of SOD was expressed as units per milligram protein.

Determination of glutathione peroxidase (GSHPx): The activity of GSHPx in brain homogenate was determined indirectly by measuring the yellow colored 5-thio-2-nitrobenzoic acid which produced from the reaction of glutathione (GSH) and DTNB (Eyer and Podhradský, 1986). Standard GSHPx concentrations ranging between 1-100 units $\mathrm{ml}^{-1}$ were used as calibration curve. The activity was normalized with protein concentration and expressed as units per milligram protein.

Determination of Catalase (CAT): Catalase activity was measured by recording the rate of $\mathrm{H}_{2} \mathrm{O}_{2}$ reduction (Goldblith and Proctor, 1950) The brain homogenate sample or standard CAT concentrations ranging between 0-100 units. $\mathrm{ml}^{-1}$ were allowed to react with $0.01 \mathrm{~N} \mathrm{H}_{2} \mathrm{O}_{2}$ and stopped the reaction with $5 \mathrm{~N}$ sulfuric acid solution. $5 \mathrm{mM}$ Potassium permanganate was added to perform the reaction with excess peroxide and the excess permanganate from the reaction with peroxide was determined photometrically at $515 \mathrm{~nm}$. The standard curve was plotted as the A515 nm against the catalase activity. The data was expressed as units of catalase per mg protein.

Experimental protocols: Animals were divided into 6 groups; each group consisted of 8 animals as following:

- Group I: Sham operation group, animals were orally administered with $1 \%$ carboxymethylcellulose and received sham operation which used to determine the effect of surgery

- $\quad$ Group II: Vehicle treated group, animals were orally administered with $1 \%$ carboxymethylcellulose and served as control group

- Group III: Vitamin C, a well known antioxidant which was used as positive control was orally administered to the animals at dose of $250 \mathrm{mg} \mathrm{kg}^{-1}$ body weight
- Group IV-VI: M. oleifera extract treated groups which were orally given the leaves extract at doses of 100,200 and $400 \mathrm{mg} \mathrm{kg}^{-1}$ body weight

All animals were orally given the assigned substances for 14 days period, then, the right middle cerebral artery were performed in animals of group IIVI. $24 \mathrm{~h}$ after the induction, animals were sacrificed for determining the infarction volume $(n=4 /$ group $)$ and biochemical assays ( $n=4$ /group).

Statistical analysis: All data were presented as mean \pm SEM value. Statistical analysis was operated using SPSS ${ }^{\circledR}$ (v. 17.0 for Window ${ }^{\circledR}$ ). Statistical significant of data were performed using one way Analysis Of Variance (ANOVA) followed by LSD post hoc test for multiple comparison. The statistical significant level was set at p-value $<0.05$.

\section{RESULTS}

Effect of $M$. oleifera leaves extract on brain infarction: We had determined the brain infarct volume in rats subjected to the right middle cerebral artery occlusion. Our results in Fig. 1 clearly demonstrated that rats which receive sham operation showed no infarction volume whereas rats which received the occlusion of right middle cerebral artery showed the significant increase in infarction volume of both cortex and subcortex ( $\mathrm{p}$-value $<0.001$ all; compared with vehicle plus sham operation). Rats which subjected vitamin $\mathrm{C}$ significantly decreased the infarction volume both in cortex and subcortex (pvalue<0.01 all; compared with vehicle plus MCAO). Interestingly, it was found that M.oleifera leaves extract at doses of 100,200 and $400 \mathrm{mg} \mathrm{kg}^{-1} \mathrm{BW}$ could decrease brain infarction volume in cortex (pvalue $<0.01,0.05$ and 01.05 respectively; compared with vehicle plus MCAO) and subcortex (pvalue $<0.01,0.05$ and 0.01 respectively; compared with vehicle plus MCAO).

Effect of $M$. oleifera leaves extract on the alteration of malondialdehyde level: The current data in Fig. 2 revealed that rats which were induced MCAO significantly increased MDA level in cortex, hippocampus and striatum (p-value $<0.01,0.01$ and 0.05 respectively; compared with vehicle plus sham operation). Vitamin $\mathrm{C}$ treated group reversed the elevation of MDA induced by MCAO in cerebral cortex, hippocampus and striatum (p-value $<0.01$, 0.01 and 0.05 respectively; compared with vehicle plus MCAO). 


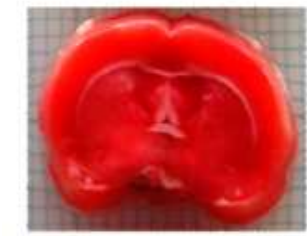

Vehicle+Sham operation

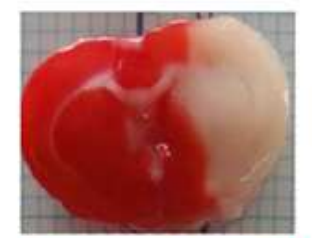

M. oleifera $100+\mathrm{MCAO}$

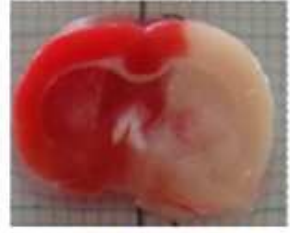

Vehicle+MCAO

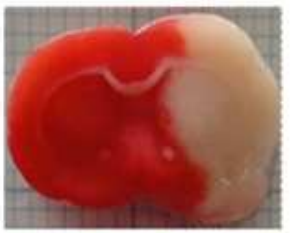

M. oleifera $200+\mathrm{MCAO}$

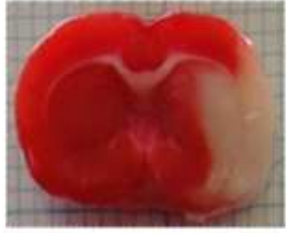

Vitamin $\mathrm{C}+\mathrm{MCAO}$

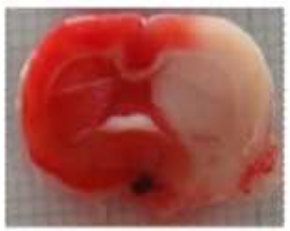

M. oleifera $400+\mathrm{MCAO}$

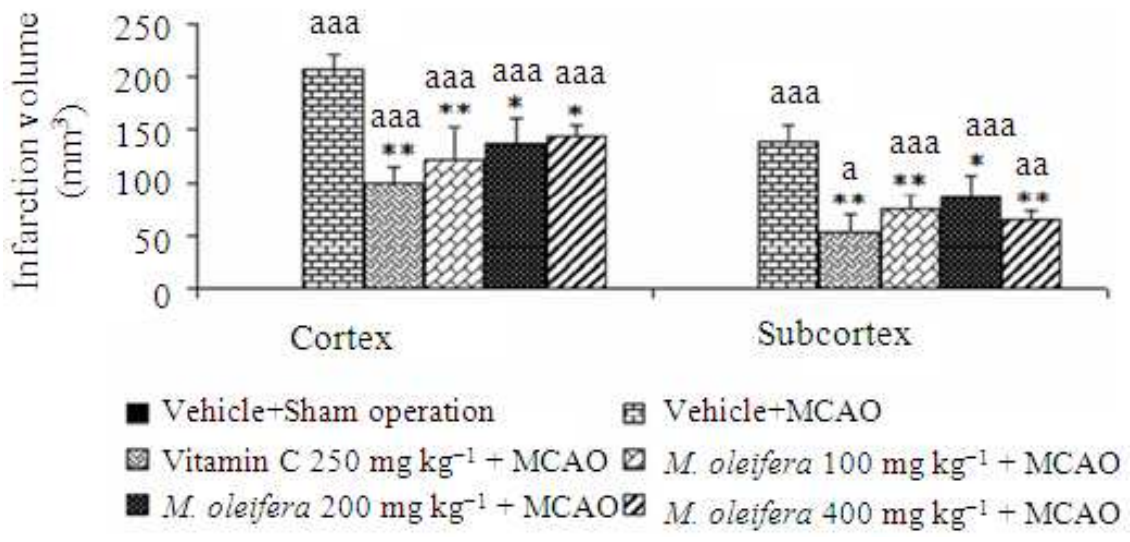

Fig. 1: The effect of Vitamin C, M. oleifera extract at the doses of 100, 200 and $400 \mathrm{mg} \mathrm{kg}^{-1} \mathrm{BW}$ on the brain infarction volume. The coronal brain sections were determined using TTC staining. The data value are expressed as mean \pm S.E.M. ${ }^{a}$ : p-value $<.05,{ }^{\text {aa }}$ : p-value $<0.01,{ }^{\text {aaa }}$ : p-value $<0.001$; compared with vehicle plus sham operation. *: p-value $<0.05, * *$ : p-value $<0.01$; compared with vehicle plus MCAO

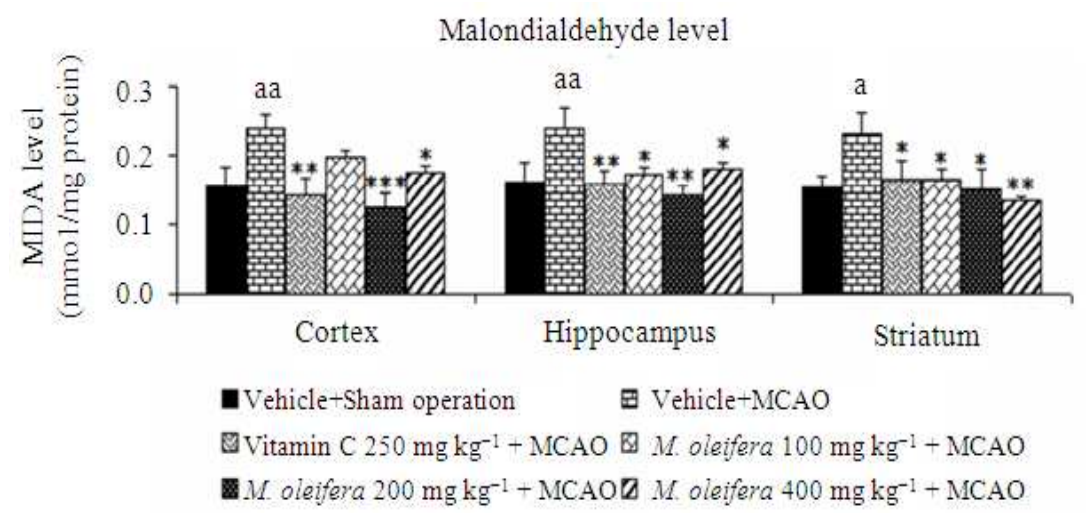

Fig. 2: The effect of Vitamin C, M. oleifera extract at the doses of 100, 200 and $400 \mathrm{mg} \mathrm{kg}^{-1} \mathrm{BW}$ on the alteration of Malondialdehyde (MDA), oxidative stress marker in cortex, hippocampus and striatum. The data value are expressed as mean \pm S.E.M. ${ }^{\text {a }}$ p-value $<0.05,{ }^{\text {aa }}$ : p-value $<0.01$; compared with vehicle plus sham operation group. $*$ : p-value $<0.05, * *$ : p-value $<0.01$, *** p-value $<0.001$; compared with vehicle plus MCAO 


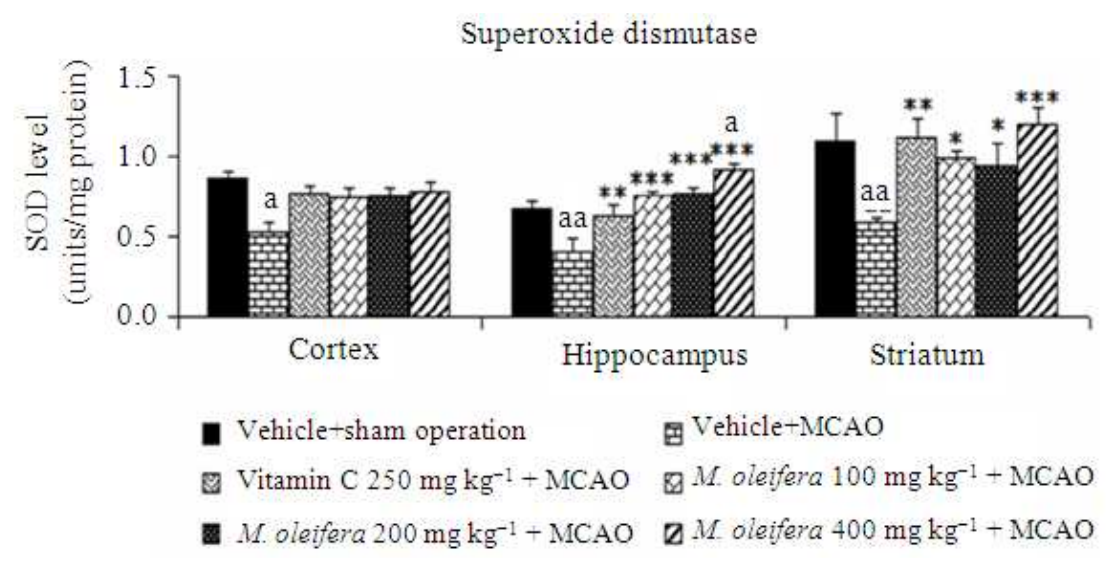

Fig. 3: The effect of Vitamin C, M. oleifera extract at the doses of 100, 200 and $400 \mathrm{mg} \mathrm{kg}^{-1} \mathrm{BW}$ on the activity of Superoxide Dismutase (SOD) in cerebral cortex, hippocampus and striatum. The data are expressed as mean \pm S.E.M. ${ }^{a}:$ p-value $<0.05,{ }^{\text {aa }}$ : p-value $<0.01$ as compared with vehicle plus sham operation group. *: pvalue $<0.05, * *: \mathrm{p}$-value $<0.01, * * *$ : $\mathrm{p}$-value $<0.001$; compared with vehicle plus MCAO

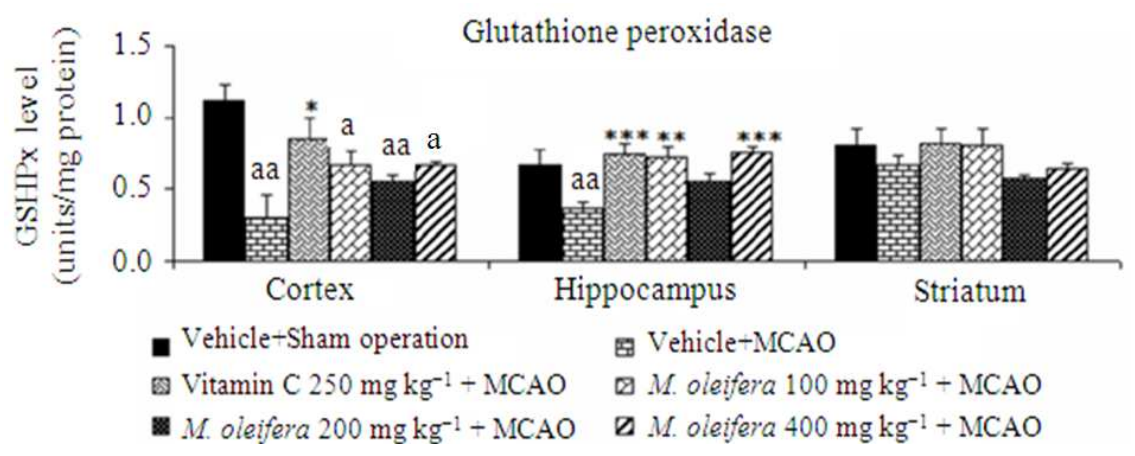

Fig. 4: The effect of Vitamin C, M. oleifera extract at the doses of 100, 200 and $400 \mathrm{mg} \mathrm{kg}^{-1} \mathrm{BW}$ on the activity of Glutathione Peroxidase (GSHPx) in cerebral cortex, hippocampus and striatum. The data are expressed as mean \pm S.E.M. ${ }^{a}:$ p-value $<0.05,{ }^{\text {aa }}$ : p-value $<0.01$; compared with vehicle plus sham operation. $*$ : p-value $<$ $0.05, * *$ : p-value $<0.01, * * *$ : p-value $<0.001$; compared with vehicle plus MCAO

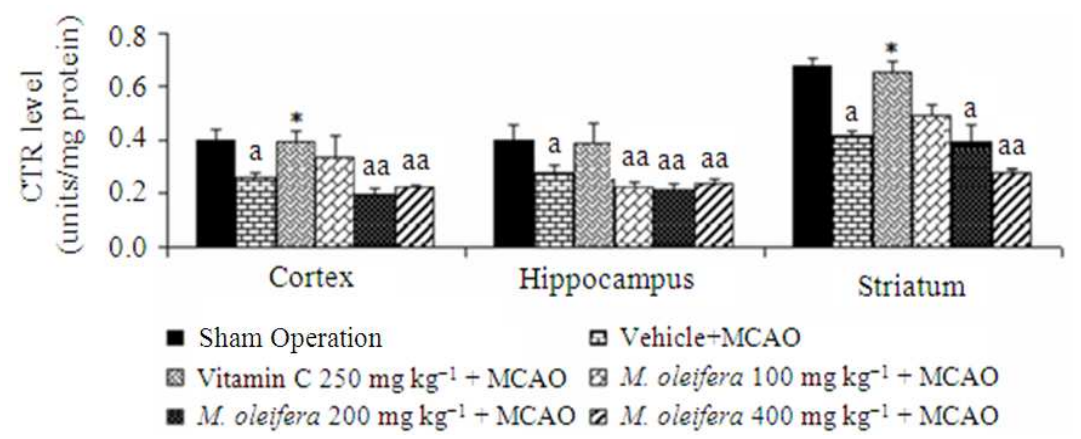

Fig. 5: The effect of Vitamin C, M. oleifera extract at the doses of 100, 200 and $400 \mathrm{mg} \mathrm{kg}^{-1}$ BW on the activity of Catalase (CAT) in cerebral cortex, hippocampus and striatum. The data are expressed as mean \pm S.E.M. ${ }^{a}:$ pvalue $<0.05,{ }^{\text {aa }}$ : p-value $<0.01$; compared with vehicle plus sham operation. *: p-value $<0.05$; compared with vehicle plus MCAO 
Effect of $M$. oleifera leaves extract on the antioxidant enzymes: The effect of $M$. oleifera on the alteration of SOD was shown in Fig. 3. The results demonstrated that MCAO significantly decreased SOD activity in cerebral cortex, hippocampus and striatum (p-value $<0.05,0.01$ and 0.01 respectively; compared with vehicle plus sham operation). Vitamin $\mathrm{C}$ treated group showed the significant enhanced SOD activity in hippocampus and striatum (p-value $<0.01$ all; compared with vehicle plus MCAO). All doses of $M$. oleifera treated groups also enhanced SOD activity in hippocampus ( $p$-value $<0.001$ all; compared with vehicle plus MCAO) and striatum (p-value<0.05, 0.05 and 0.001 respectively; compared with vehicle plus MCAO). Surprisingly, the high dose of extract appeared to increase SOD activity in hippocampus greater than vitamin $\mathrm{C}$ treated group. Figure 4 showed that MCAO could decrease GSHPx activity in both cerebral cortex and hippocampus (p-value $<0.01$ all; compared with vehicle plus sham operation). Vitamin $\mathrm{C}$ treated groups significantly revered the reduction of GSHPx activity in cerebral cortex and hippocampus ( $p-$ value $<0.05$ and 0.001 respectively; compared with vehicle plus MCAO). The low and high doses treated groups also reversed the reduction of GSHPx activity in the mentioned area ( $\mathrm{p}$-value<0.01 and 0.001 respectively; compared with vehicle plus MCAO). No other significant changes were observed in cerebral cortex and striatum. It was also found that MCAO could also decrease the activity of CAT in cerebral cortex, hippocampus and striatum as shown in Fig. 5 (p-value $<0.05$ all; compared with vehicle plus sham operation). Vitamin $\mathrm{C}$ treated group could enhance CAT activity in cereral cortex and striatum (pvalue<0.05 all; compared with vehicle plus MCAO) but no significant changes were observed in M.oleifera treated groups.

The medium and high doses of M.oleifera also significantly reversed the enhanced MDA level in cerebral cortex (p-value $<0.001,0.05$ respectively; compared with vehicle plus MCAO), hippocampus (p-value $<0.01,0.05$ respectively; compared with vehicle plus MCAO) and striatum (p-value<0.05, 0.01 respectively; compared with vehicle plus MCAO). The low dose of extract could mitigate the elevation of MDA level in hippocampus and striatum (p-value $<0.05$ all; compared with vehicle plus MCAO).

\section{DISCUSSION}

Our study have demonstrated the neuroprotective potential of hydroalcoholic extract of M.oleifera leaves extract against ischemia induced oxidative stress as well as histopathological alteration.
It is well documented that focal MCAO gives rise to neurological and histopathological abnormalities in some brain areas. These abnormalities have been reported to be associated with Reactive Oxygen Species (ROS), which react with cellular macromolecules such as lipids, proteins and nucleic acids resulting in oxidative damage of neurons (Negishi et al., 2001). In this study, our results have demonstrated the reduction of SOD and CAT in cerebral cortex, hippocampus and striatum whereas the reduction of GSHPx was observed only in cerebral cortex and hippocampus of cerebral ischemic rats. The decreased enzymes activities in turn enhanced the excess reactive oxygen species resulting in the increased oxidative damage reflecting by the elevation of MDA in cerebral cortex, hippocampus and striatum leading to neurodegeneration and brain infarction. It was found that M.oleifera leaves extract markedly decreased MDA level in all areas mentioned earlier and decreased brain infarction. The extract could enhance the activity of SOD both in hippocampus and striatum whereas the elevation of GSHPx enzyme activity was observed only in hippocampus. Unfortunately no changes of any enzymes just mentioned were observed in cerebral cortex. Therefore, the reduction of MDA level in this area might occur via the reduction of oxidative stress formation.

Although the determination of active ingredient responsible for the neuroprotective effect of this plant extract is beyond the scope of this study, we do suggest that the polyphenolic compound in the extract might play a vital role. It has been reported that plant polyphenols provide protection against neurodegenerative changes associated with cerebral ischemia (Simonyi et al., 2005). Moreover, this compound could also reduce the infarct volume, prevented motor impairment and inhibited lipid peroxidation (Sinha et al., 2002). Based on the effect of polyphenolic compounds just mentioned, it was also possible that the neuroprotective effect of the plant extract was associated with these compounds. Though our results suggested that the neuroprotective effect of M.oleifera leaves extract occurred partly via the decreased oxidative stress, other mechanisms such as the decreased apoptosis and intracellular calcium which also played the crucial roles on neurodgeneration and brain damage in cerebral ischemia still could not be cut off.

\section{CONCLUSION}

In conclusion, this study is the first study to demonstrate the neuroprotective effect against focal cerebral ischemia of M.oleifera leaves. It suggests that M.oleifera may be served as natural resource for developing neuroprotectant against focal cerebral 
ischemia. However, the precise underlying mechanism and possible active ingredient are still required further study.

\section{ACKNOWLEDGEMENT}

This study was supported by the Higher Education Research Promotion and National Research University Project of Thailand, Office of the Higher Education Commission, through the Food and Functional Food Research Cluster of Khon Kaen University, Integrative Complimentary Alternative Medicine Research and Development Group, Khon Kaen University. Moreover, we also would like to express our sincere thank to Associate Professor Bungorn Sripanidkulchai, Director of Center for Research and Development of Herbal Health Product, Khon Kaen University for her kindly management through Functional Food Cluster.

\section{REFERENCES}

Kumar, N.A. and L. Pari, 2003. Antioxidant action of Moringa Oleifera lam. (Drumstick) against antitubercular drugs induced lipid peroxidation in rats. J. Med. Food, 6: 255-259. DOI: 10.1089/10966200360716670

Caceres, A., A. Saravia, S. Rizzo, L. Zabala and E.D. Leon et al., 1992. pharmacologic properties of Moringa Oleifera. 2: Screening for antispasmodic, antiinflammatory and diuretic activity. J. Ethnopharmacol., 36: 233-237. PMID: 1434682

Eyer, P. and D. Podhradský, 1986. Evaluation of the micromethod for determination of glutathione using enzymatic cycling and Ellman's reagent. Anal. Biochem., 153: 57-66. DOI: 10.1016/00032697(86)90061-8

Faizi, S., B.S. Siddiqui, R. Saleem, S. Siddiqui and K. Aftab et al., 1995. Fully acetylated carbamate and hypotensive thiocarbamate glycosides from Moringa Oleifera. Phytochemistry, 38: 957-963. DOI: 10.1016/0031-9422(94)00729-D

Feigin, V.L., 2007. Stroke in developing countries: can the epidemic be stopped and outcomes improved? Lancet Neurol., 6: 94-97. DOI: 10.1016/S14744422(07)70007-8

Goldblith, S.A. and B.E. Proctor, 1950. Photometric determination of catalase activity. J. Biol. Chem., 187: 705-709.

Kakkar, P., B. Das and P.N. Viswanathan, 1984. A modified spectrophotometric assay of superoxide dismutase. Indian J. Biochem. Biophys., 21: 130132. PMID: 6490072
Lowry, O.H., N.J. Rosebrough, A.L. Farr and R.J. Randall, 1951. Protein measurement with the folin phenol reagent. J. Biol. Chem., 193: 265-275.

Margaill, I., M. Plotkine and D. Leroue, 2005. Antioxidant strategies in the treatment of stroke. Free Radic Biol. Med., 39: 429-43. DOI: 10.1016/j.freeradbiomed.2005.05.003

Mohan, M., N. Kaul, A. Punekar, R. Girnar and P. Junnare et al., 2005. Nootropic activity of Moringa Oleifera leaves. J. Nat. Remedies.,

Negishi, H., K. Ikeda, Y. Nara and Y. Yamori, 2001. Increased hydroxyl radicals in the hippocampus of stroke-prone spontaneously hypertensive rats during transient ischemia and recirculation. Neurosci. Lett., 306: 206-208. DOI: 10.1016/S0304-3940(01)01893-6

Simonyi, A., Q. Wang, R.L. Miller, M. Yusof and P.B. Shelat et al., 2005. Polyphenols in cerebral ischemia: Novel targets for neuroprotection. Mol. Neurobiol., 31: 135-147. DOI: 10.1385/MN:31:1-3:135

Sinha, K., G. Chaudhary and Y.K. Gupta, 2002. Protective effect of resveratrol against oxidative stress in middle cerebral artery occlusion model of stroke in rats. Life Sci., 71: 655-665. DOI: 10.1016/S0024-3205(02)01691-0

Sreelatha, S. and P.R. Padma, 2011. Modulatory effects of Moringa Oleifera extracts against hydrogen peroxide-induced cytotoxicity and oxidative damage. Hum. Exp. Toxicol., 30: 1359-1368. DOI: 10.1177/0960327110391385

Sudha, P., S.M. Asdaq, S.S. Dhamingi and G.K. Chandrakala, 2010. Immunomodulatory activity of methanolic leaf extract of Moringa Oleifera in animals. Indian J. Physiol Pharmacol., 54: 133-140.

Tong-Un, T., P. Wannanon, J. Wattanathorn and W. Phachonpai, 2010. Cognitive-enhancing and antioxidant activities of quercitin liposomes in aminal model of Alzheimer's disease. Online J. Biol. Sci., 10: 84-91. DOI: 10.3844/ojbsci.2010.84.91

Warner, D.S., H. Sheng and I. Batinic-Haberle, 2004. Oxidants, antioxidants and the ischemic brain. J. Exp. Biol., 207: 3221-31. PMID: 15299043

Wattanathorn, J., J. Jittiwat, T. Tongun, S. Muchimapura and I. Kornkanok, 2011. Zingiber officinale mitigates brain damage and improves memory impairment in focal cerebral ischemic rat. Evid. Based Complement Alternat Med., 2011: 18. DOI: $10.1155 / 2011 / 429505$ 